\title{
Remembering Richard C. Nelson: An Introduction
}

Richard (Dick) C. Nelson was the founding editor of the International Journal of Sport Biomechanics. Its first issue was published in 1985, and in 1991 it became the Journal of Applied Biomechanics. Dr. Nelson passed away on Tuesday, December 22,2020 at the age of 88 years. This special section is designed to reflect many of Dr. Nelson's contributions to biomechanics. Dr. Nelson was a seminal figure in biomechanics who, in addition to starting a journal, made contributions across many domains. The special section includes contributions from some of his former graduate students and from international colleagues. There are perspectives, which reflect personal viewpoints on different aspects of Dr. Nelson's contributions, along with articles authored by his former students.

The international development of biomechanics was an important focus of Dr. Nelson, some of his contributions in this area are highlighted in the perspective of Benno Nigg (University of Calgary). Vladimir Zatsiorsky (The Penn State University) followed Dr. Nelson as the Director of the Penn State Biomechanics Lab in the 1990s, but they first met in 1971. His perspective highlights how Dr. Nelson contributed to the development of modern biomechanics, how he negotiated soviet era bureaucracy, and once again how he worked diligently to develop biomechanics as an international field of study.

Dr. Nelson advised 66 master's students and 33 doctoral students, as well as serving on many other student committees. Bob Gregor (UCLA, The Georgia Institute of Technology, USC, and the University of Nevada, Las Vegas) commenced his graduate studies with Dr. Nelson in 1971. Bob followed Dr. Nelson as Editor-in-Chief of the International Journal of Sport Biomechan$i c s$, and oversaw its renaming to the Journal of Applied Biomechanics. Bob provides lessons learnt from Dr. Nelson as a mentor, as well as focusing on his work for the International Society of Biomechanics, and the recording and subsequent analysis of athlete performances as a member of the IOC Medical Commission at several Olympic Games.

Dr. Nelson was a strong advocate of the collection of athlete performance at major competitions, one of his earliest graduate students, Doris Miller (Western University), actively contributed to some of these efforts. She describes the evolving nature of data collection, and the issues that arise from competition site data collection.

In supervising the work of graduate students, an adviser produces academic children, students of these students produce academic grandchildren and so on. Richard Nelson has a large population of academic children (just under 100), and consequently many academic grandchildren and great grandchildren. Robert Norman (Nelson academic child) and his colleagues from the University of Waterloo, Stuart McGill (academic grandchild), and James Potvin (academic great grandchild), describe the important influence of Richard Nelson on the development of the teaching and research programs at the University of Waterloo.
Dr. Nelson's lab was situated in a water tower, a building that had the look of an observatory, but with the top being a dome containing water rather than a telescope. When Robert Shapiro (University of Kentucky) first entered that lab, it is unlikely he thought that some of his work would end up in the baseball hall of fame. His perspective explains Dr. Nelson's philosophy on the advising of students.

As has been reflected in other perspectives Dr. Nelson was a proponent of the internationalization of biomechanics in general. The perspective of Mitsumasa Miyashita (University of Tokyo) highlights the impact that Dr. Nelson had on the development of biomechanics in Japan.

In the latter half of Dr. Nelson's career he became involved in forensic biomechanics, focusing on the biomechanical determination of whether an accident was the cause of an alleged injury. The perspective provided by Kathryn Dainty Davis (Exponent, Inc) indicates how Dr. Nelson's form of mentorship provided her with the background to enter forensic biomechanics.

The perspectives end with the reflections of James Walton on a career in biomechanics, specifically innovations in 3-dimensional photogrammetry, and how it was influenced by the early facilitation he received from Dr. Nelson.

This special section concludes with 3 research papers contributed by former graduate students of Dr. Nelson. Jill McNitt-Gray (University of Southern California) and her co-authors examine aspects of the control of diving, an activity on which Dr. Nelson published (e.g., Miller et $\mathrm{al}^{1}$ ). Gary Heise (University of Northern Colorado) and co-authors present a paper on the dynamic postural stability post anterior cruciate ligament reconstruction surgery. Of note in this paper is that all the other authors are Nelson academic grandchildren. Finally, Young-Hoo Kwon (Texas Woman's University) and his co-authors present a paper on the mechanics of golf. Dr. Nelson was fond of a round of golf so would have found this paper interesting; although, he often opined that the game should be won by the person who completed the round in the shortest time, and not in the fewest number of strokes.

Dr. Nelson had a career which had influence across a number of domains. It is our hope that these perspectives and research papers not only give some impression of the breadth of his contributions, but also provide insights for others to follow.

\section{John H. Challis and Stephen J. Piazza, Biomechanics Laboratory, The Pennsylvania State University, University Park, PA, USA}

\section{Reference}

1. Miller DI, Hennig EM, Pizzimenti MA, Jones IC, Nelson RC. Kinetic and kinematic characteristics of 10-m platform performances of elite divers: I. Back takeoffs. Int J Sport Biomech. 1989;5(1):60-88. doi:10. 1123/ijsb.5.1.60 